\title{
Effect of anti-PcrV antibody in a murine chronic airway Pseudomonas aeruginosa infection model
}

\author{
Y. Imamura*, , K. Yanagihara*, , Y. Fukuda*, Y. Kaneko*, M. Seki*, K. Izumikawa*, \\ Y. Miyazaki*, Y. Hirakata*, T. Sawa*, J.P. Wiener-Kronish\# and S. Kohno*
}

\begin{abstract}
Pseudomonas aeruginosa is one of the most important pathogens in patients with chronic airway conditions, such as cystic fibrosis and diffuse panbronchiolitis. Type III secretion system-mediated virulence factors contribute to the lung damage in chronic $P$. aeruginosa infection.

The effects of the anti-PcrV immunoglobulin (Ig)G, which blocks the type III secretion system, were evaluated in a mouse model of chronic $P$. aeruginosa infection. On bacteriological examination, anti-PcrV IgG showed no bactericidal effects. On bronchoalveolar lavage fluid (BALF) analysis, total cell number and neutrophil ratios in the anti-PcrV IgG-treated groups were lower than those in the control group. In addition, macrophage inflammatory protein-2, tumour necrosis factor- $\alpha$, and interleukin- $\beta$ concentrations in BALF were lower in the anti-PcrV IgG-treated groups when compared with controls. Plasma anti-PcrV IgG titre was elevated after administration of anti-PcrV IgG. Although plasma titre decreased gradually, a significant concentration was maintained during the experimental period.

These data suggest that anti-PcrV immunoglobulin G reduces the inflammatory reaction caused by chronic Pseudomonas aeruginosa respiratory infection and may be useful in treating respiratory diseases.
\end{abstract}

KEYWORDS: Anti-PcrV antibody, chronic airway infection, Pseudomonas aeruginosa, type III secretion system

$P$

seudomonas aeruginosa is an opportunistic pathogen associated with hospitalacquired pneumonia and chronic airway infection in patients with chronic respiratory conditions, such as cystic fibrosis and diffuse panbronchiolitis. Even if strong antimicrobial chemotherapy is performed, it is very difficult to completely eradicate $P$. aeruginosa, and chronic infection can cause lung tissue damage that eventually leads to severe respiratory failure.

P. aeruginosa produces numerous extracellular virulence factors, which contribute to its pathogenesis in chronic infection. These virulence factors are secreted into the surrounding extracellular environment via the type I, II or III protein secretion systems. Toxins such as exoenzyme S, T, U and Y are directly transported into eukaryotic cells through the type III secretion system [1-4]. Expression of these toxins causes epithelial injury and inhibits the host immune response [3, 5-7]. PcrV is homologous to the Vantigen $(\mathrm{LcrV})$ found on the surface of Yersinia and appears to be an integral component of the translocation process [7]. In a previous study, it was shown that administration of anti-PcrV antibody $(\mathrm{Ab})$ was able to block the translocation of $P$. aeruginosa type III-secreted toxins [7]. Anti-PcrV immunoglobulin (Ig)G also exerted therapeutic effects, reducing septic shock and mortality in an animal model of acute $P$. aeruginosa infection $[7,8]$. However, the efficacy of anti-PcrV IgG in chronic $P$. aeruginosa infection has not yet been clarified. In the present study, the effects of anti-PcrV IgG in a murine model of chronic $P$. aeruginosa respiratory infection were investigated.

\section{MATERIALS AND METHODS Laboratory animals}

Five-week-old, male, ddY, specific-pathogen-free mice were purchased from Shizuoka Agricultural Cooperative Association Laboratory Animals (Shizuoka, Japan). All animals were housed in a pathogen-free environment and received sterile food and water in the Laboratory Animal Center for Biomedical Science at Nagasaki University
AFFILIATIONS

*Second Dept of Internal Medicine, Nagasaki University School of Medicine, Nagasaki, Japan. \#Dept of Anesthesia and Critical Care, University of California, San Francisco, CA, USA.

"Both authors contributed equally to this study.

\section{CORRESPONDENCE}

K. Yanagihara

The Second Dept of Internal Medicine Nagasaki University School of Medicine 1-7-1 Sakamoto

Nagasaki 852-8501

Japan

Fax: 81958497285

E-mail: kyana-ngs@umin.ac.jp

Received:

November 132006

Accepted after revision:

January 152007

SUPPORT STATEMENT

This study was supported by grants for scientific research (1759079600) from the Ministry of Education, Culture, Sports, Science and Technology of Japan. Part of the information in this manuscript was presented at the 45th Interscience Conference on Antimicrobial Agents and Chemotherapy (Washington, DC, USA)

STATEMENT OF INTEREST

None declared.

European Respiratory Journal Print ISSN 0903-1936 Online ISSN 1399-3003 
(Nagasaki, Japan). The experimental protocols were approved by the Ethics Review Committee for Animal Experimentation at the current authors' institution (Nagasaki).

\section{Bacterial strains and culture conditions}

Nonmucoid P. aeruginosa strain 6073, a human corneal isolate, was used in the present study [9]. The strain was serogroup O11 and was characterised as cytotoxic [9]. For each experiment, strains were plated from $10 \%$ skimmed milk (Yukijirushi Co., Tokyo, Japan) stocks maintained at $-70^{\circ} \mathrm{C}$. All cultures were grown at $37^{\circ} \mathrm{C}$, and liquid cultures were shaken at $250 \mathrm{rpm}$.

\section{Murine model of chronic P. aeruginosa respiratory infection}

Chronic airway infection was induced in mice using the method described by YANAGIHARA et al. [10]. Disposable, sterile, plastic cut-down i.v. catheters ( $3 \mathrm{Fr}, 1.0 \mathrm{~mm}$ diameter; Atom Co., Tokyo, Japan) were used for tracheal intubation. The tubes were $3.0 \mathrm{~mm}$ in length, with slits at the proximal end in order to prevent blockage by oral secretions. Bacteria were grown overnight from frozen stocks on Trypticase Soy agar plates. Bacteria were suspended in sterile saline in order to achieve an optical density of 0.2 at $660 \mathrm{~nm}$. The plastic tube was then immersed in bacterial saline suspension for 3 days at $37^{\circ} \mathrm{C}$. The intubation procedure was performed under pentobarbital anaesthesia. The blunted end of the inner needle of an i.v. catheter (Angiocath; Becton Dickinson Vascular Access, Sandy, UT, USA) was inserted through the oral cavity with the outer sheath and attached tube at the tip. The tube was advanced through the vocal cords into the trachea. The inner needle was then removed, and the outer sheath was gently pushed in order to place the plastic tube into the main bronchus. Infection was restricted to the lung and no animals died as a result of subsequent infection.

\section{Antibody administration}

The polyclonal rabbit-derived anti-PcrV $\mathrm{Ab}$ and pre-immune serum-derived control $\mathrm{Ab}$ used in the present study were prepared and stored at $-80^{\circ} \mathrm{C}$, as previously reported [8]. The mice were separated into three groups; each group received i.p. administration of control IgG on day 7 and day 14, anti-PcrV IgG on day 7 or anti-PcrV IgG on day 7 and day 14 .

\section{Bacteriological examination}

Five mice from each group were sacrificed on day 21 after inoculation. The tube was removed and the lungs were excised under aseptic conditions. The lungs were homogenised in $1.0 \mathrm{~mL}$ of PBS and were cultured quantitatively by serial dilution on Pseudomonas isolation agar.

\section{Bronchoalveolar lavage and cytokine ELISA}

Bronchoalveolar lavage (BAL) was performed as described previously [11]. Briefly, mice were sacrificed on day 21 after inoculation. The chest was opened to expose the lungs and a disposable sterile plastic cutdown i.v. catheter was inserted into the trachea. BAL was performed three times sequentially using $1 \mathrm{~mL}$ saline each time; the recovered fluid fractions were pooled for each animal. Fluid recovery was routinely $\geqslant 90 \%$. Leukocytes in the BAL fluid (BALF) samples obtained from each mouse were washed and counted with a haemocytometer. For differential cell counts, cells were centrifuged at $1,000 \times g$ for $1 \mathrm{~min}$ onto slides, which were then stained with May-Giemsa stain. Differential cell counts were performed by counting 100 cells. Concentrations of macrophage inflammatory protein (MIP)-2, tumour necrosis factor (TNF)- $\alpha$ and interleukin (IL)- $1 \beta$ in BALF were assayed using mouse cytokine ELISA test kits ( $\mathrm{R}$ \& $\mathrm{D}$ Systems, Minneapolis, MN, USA).

\section{Plasma anti-PcrV IgG titre}

Serial changes in anti-PcrV titres in mouse plasma were determined. Immunoplates for ELISA were coated with $\mathrm{rPcrV}$ protein $\left(10 \mathrm{~g} \cdot \mathrm{mL}^{-1}\right)$ and were incubated overnight at $4^{\circ} \mathrm{C}$. Plasma samples were added to the plates, followed by application of anti-rabbit monoclonal IgG conjugated with alkaline phosphatase (Sigma-Aldrich, St. Louis, MO, USA) as a secondary Ab. Plates were washed 2-6 times with PBS/Tween 20 after addition of each $\mathrm{Ab}$. Optical density was measured at $405 \mathrm{~nm}$ after adding phosphatase substrates (Sigma 104; Sigma-Aldrich). IgG concentration in each sample was calculated by comparing the absorbance to a standard curve constructed using anti-PcrV IgG solution.

\section{Statistical analysis}

All data are expressed as mean \pm SEM. Differences between groups were examined for statistical significance using an unpaired t-test. A p-value $<0.05$ denoted the presence of a statistically significant difference.

\section{RESULTS}

\section{Bacteriological examination}

In order to determine the bactericidal effects of anti-PcrV IgG against $P$. aeruginosa, viable bacterial numbers in the lungs were evaluated. The numbers of viable bacteria in the lungs of mice 14 days after inoculation were $4.80 \pm 0.46 \log _{10}$ colonyforming units (CFU) lung $^{-1}$ in the control IgG-treated group, $5.46 \pm 0.47 \log _{10} \mathrm{CFU} \cdot \mathrm{lung}^{-1}$ in the anti-PcrV IgG-treated once group, and $5.38 \pm 0.43 \log _{10} \mathrm{CFU}$ lung $^{-1}$ in the anti-PcrV IgGtreated twice group. The differences in the number of viable bacteria in the lung among the three groups were not statistically significant. Anti-PcrV IgG did not exhibit any bactericidal effects against $P$. aeruginosa strain 6073 .

\section{Effects of anti-PcrV IgG on cell numbers in BALF}

The effects of anti-PcrV IgG against inflammatory changes induced by chronic $P$. aeruginosa infection in the lung were examined in BALF on day 21 after inoculation. The total numbers of cells in the once-treated anti-PcrV IgG group and the twice-treated anti-PcrV IgG group were significantly lower than in the control IgG-treated group (table 1). The ratio of neutrophils/total cells in the once-treated anti-PcrV IgG group and the twice-treated anti-PcrV IgG group was significantly lower than in the control IgG-treated group (table 1).

\section{Cytokine levels in BALF}

In order to further explore the effects of anti-PcrV IgG in $P$. aeruginosa chronic respiratory infection, cytokine levels in BALF were analysed. MIP-2 (fig. 1a), IL- $\beta$ (fig. 1b) and TNF$\alpha$ (fig. 1c) concentrations were significantly lower in the twicetreated anti-PcrV IgG group when compared with the control IgG-treated group. 


\begin{tabular}{|c|c|c|c|}
\hline \multirow[t]{2}{*}{ Cell type } & \multirow[t]{2}{*}{ Control IgG } & \multicolumn{2}{|c|}{ Anti-PcrV IgG } \\
\hline & & Treated once & Treated twice \\
\hline Total $10^{5} \cdot \mathrm{mL}^{-1}$ & $4.92 \pm 0.39$ & $2.23 \pm 0.21^{\circ}$ & $2.29 \pm 0.42^{\circ}$ \\
\hline Neutrophils $10^{5} \cdot \mathrm{mL}^{-1}$ & $2.97 \pm 0.34(60.4 \pm 7.0)$ & $0.76 \pm 0.10(34.3 \pm 4.6)^{\circ}$ & $0.35 \pm 0.13(15.1 \pm 5.8)^{\star *}$ \\
\hline Lymphocytes $10^{5} \cdot \mathrm{mL}^{-1}$ & $0.13 \pm 0.02(2.6 \pm 0.4)$ & $0.06 \pm 0.01(2.8 \pm 0.6)$ & $0.08 \pm 0.01(3.6 \pm 0.5)$ \\
\hline Macrophages $10^{5} \cdot \mathrm{mL}^{-1}$ & $1.85 \pm 0.32(37.5 \pm 6.5)$ & $1.41 \pm 0.10(63.3 \pm 4.7)^{\bullet}$ & $1.88 \pm 0.16(82.3 \pm 6.8)^{\star \star}$ \\
\hline
\end{tabular}

\section{Plasma anti-PcrV IgG levels}

Serial changes in plasma anti-PcrV IgG were measured. In the control IgG-treated group, the current authors were not able to detect anti-PcrV IgG during the experimental period. In the once-treated anti-PcrV IgG group (treated on day 7 after infection), plasma anti-PcrV titre was elevated on day 10 and had decreased on days 14, 17 and 21 after infection. In the twice-treated anti-PcrV IgG group (treated on days 7 and 14 after infection), plasma anti-PcrV IgG titre was elevated on day 17 after infection and had decreased slightly on day 21 after infection (fig. 2).

\section{DISCUSSION}

In the present study, it is shown that treatment with anti-PcrV IgG reduces the lung inflammation caused by chronic $P$. aeruginosa infection.

$\mathrm{PcrV}$ is an integral component of the type III secretion system. Expression of PcrV in clinical isolates of $P$. aeruginosa is associated with morbidity and mortality in infected patients [12]. Previous studies have shown that anti-PcrV IgG protects animals from acute $P$. aeruginosa infection [7, 8]. In these studies, anti-PcrV was given to animals at the same time, or soon after, inoculation. To investigate the effects of anti-PcrV $\mathrm{IgG}$ in chronic $P$. aeruginosa respiratory infection, anti-PcrV IgG was administered to mice on day 7 after infection.
BALF analysis showed that total cell number and the ratios of neutrophils in anti-PcrV IgG-treated groups were lower than that for the control group (table 1). These data indicate that the anti-PcrV IgG treatment may reduce the inflammatory response caused by $P$. aeruginosa infection. Chronic $P$. aeruginosa respiratory infection is characterised by chronic infiltration of inflammatory cells into the airways [13]. Neutrophil-dominated airway inflammation and progressive lung damage are the major causes of morbidity and mortality in chronic $P$. aeruginosa infection. The present data indicate that administration of anti-PcrV IgG is effective in reducing inflammation in chronic $P$. aeruginosa respiratory infection.

No differences in the number of viable bacteria in the lungs and in BALF (data not shown) were found between the antiPcrV IgG-treated group and the control IgG-treated group, which was expected, because anti-PcrV IgG has no direct bactericidal activity. However, anti-PcrV IgG has exhibited some bactericidal effects in an acute $P$. aeruginosa infection model $[7,8]$; thought to be due to the preservation of alveolar macrophage phagocytic function. In these studies, anti-PcrV IgG was able to inhibit bacterial proliferation in the lung. In contrast to the acute infection model, bacteria had already proliferated and colonised the airway in the present chronic infection model, thus, anti-PcrV IgG was unable to directly reduce the bacterial population. As chronic inflammation
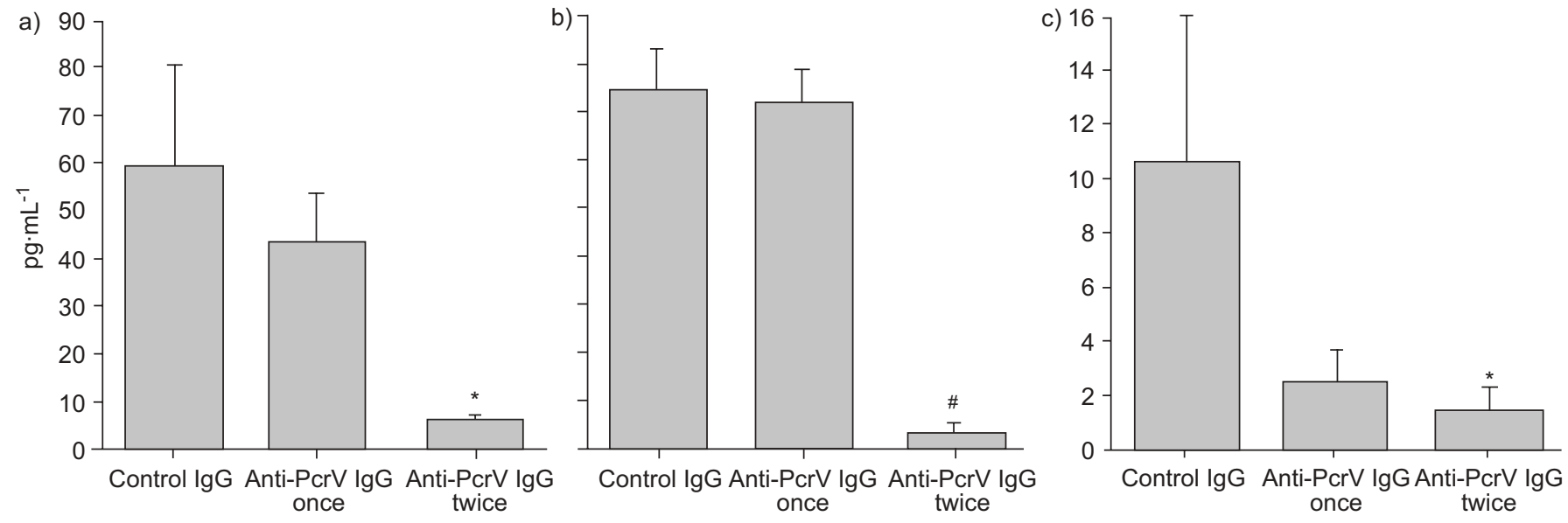

FIGURE 1. Cytokine levels in bronchoalveolar lavage fluid from eight mice on day 21 after infection. a) Macrophage inflammatory protein-2 concentration; b) interleukin- $\beta$ concentration; c) tumour necrosis factor- $\alpha$ concentration. Results are expressed as mean \pm SEM. *: $p<0.05 ;{ }^{*}: p<0.0001$ compared with control immunoglobulin (Ig)G. 


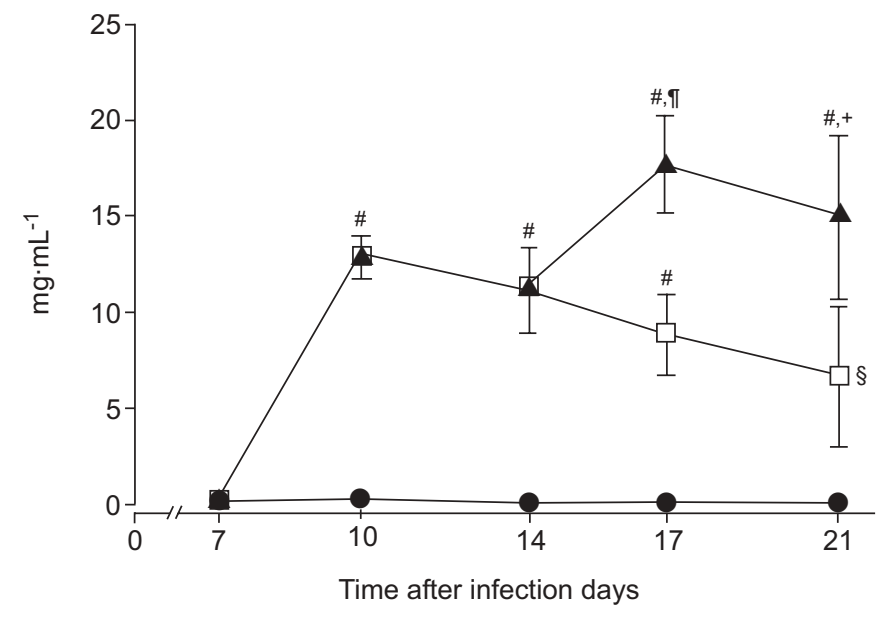

FIGURE 2. Plasma anti-PcrV titre in mice given anti-PcrV immunoglobulin (Ig)G. Results are expressed as mean \pm SEM. - : control lgG; $\square$ : once-treated antiPcrV IgG group; $\mathbf{\Lambda}$ : twice-treated anti-PcrV IgG group. ${ }^{*}: p<0.0001$ compared with control IgG; ${ }^{\varsigma}: p<0.0005$ compared with control lgG; ${ }^{\uparrow}: p<0.0001$ compared with anti - PcrV IgG (once); ${ }^{+}$: $p<0.005$ compared with anti-PcrV IgG (once)

causes airway destruction, the anti-inflammatory effects are as important as any bactericidal effects in chronic P. aeruginosa respiratory infection. Thus, administration of anti-PcrV IgG may by effective in chronic $P$. aeruginosa respiratory infection.

Pro-inflammatory cytokines play an important role in chronic $P$. aeruginosa infection as they contribute to the excessive influx of neutrophils into the airways and subsequent lung inflammation [14, 15]. On intrapulmonary cytokine analysis, MIP-2, TNF- $\alpha$ and IL-1 $\beta$ levels in the anti-PcrV IgG-treated groups were lower than those in the control group (fig. 1). Type III secretion-mediated cytotoxic proteins cause lung tissue injury and induce inflammatory reactions, such as increased pro-inflammatory cytokine production. Therefore, anti-PcrV IgG may have reduced pro-inflammatory cytokine production by preventing the lung injury caused by type III-secreted toxins.

The current authors also evaluated the changes in anti-PcrV IgG titre in mouse plasma (fig. 2). The concentration of antiPcrV was $>5 \mathrm{mg} \cdot \mathrm{mL}^{-1}$ in the once-treated group and was $>10 \mathrm{mg} \cdot \mathrm{mL}^{-1}$ in the twice-treated group. Pro-inflammatory cytokine levels were significantly lower in the anti-PcrV IgG twice-treated group, but not in the once-treated group; therefore, pro-inflammatory cytokines appear to be beneficial in maintaining serum anti-PcrV concentrations at $>10 \mathrm{mg} \cdot \mathrm{mL}^{-1}$.

In conclusion, anti-PcrV immunoglobulin G reduced excessive inflammatory reactions, such as the recruitment of inflammatory cells and the production of pro-inflammatory cytokines, caused by chronic Pseudomonas aeruginosa respiratory infection. These data suggest that treatment with anti-PcrV immunoglobulin G may be useful in chronic Pseudomonas aeruginosa respiratory infection.

\section{REFERENCES}

1 Kulich SM, Yahr TL, Mende-Mueller LM, Barbieri JT, Frank DW. Cloning the structural gene for the $49-\mathrm{kDa}$ form of exoenzyme S (exoS) from Pseudomonas aeruginosa strain 388. J Biol Chem 1994; 269: 10431-10437.

2 Yahr TL, Barbieri JT, Frank DW. Genetic relationship between the 53- and 49-kilodalton forms of exoenzyme S from Pseudomonas aeruginosa. J Bacteriol 1996; 178: 1412-1419.

3 Finck-Barbancon V, Goranson J, Zhu L, et al. ExoU expression by Pseudomonas aeruginosa correlates with acute cytotoxicity and epithelial injury. Mol Microbiol 1997; 25: 547-557.

4 Yahr TL, Vallis AJ, Hancock MK, Barbieri JT, Frank DW. ExoY, an adenylate cyclase secreted by the Pseudomonas aeruginosa type III system. Proc Natl Acad Sci USA 1998; 95: 13899-13904.

5 Kudoh I, Wiener-Kronish JP, Hashimoto S, Pittet JF, Frank D. Exoproduct secretions of Pseudomonas aeruginosa strains influence severity of alveolar epithelial injury. Am J Physiol 1994; 267: L551-L556.

6 Sawa $\mathrm{T}$, Ohara $\mathrm{M}$, Kurahashi $\mathrm{K}$, et al. In vitro cellular toxicity predicts Pseudomonas aeruginosa virulence in lung infections. Infect Immun 1998; 66: 3242-3249.

7 Sawa T, Yahr TL, Ohara M, et al. Active and passive immunization with the Pseudomonas $\mathrm{V}$ antigen protects against type III intoxication and lung injury. Nat Med 1999; 5: 392-398.

8 Shime N, Sawa T, Fujimoto J, et al. Therapeutic administration of anti-PcrV $\mathrm{F}\left(\mathrm{ab}^{\prime}\right)_{2}$ in sepsis associated with Pseudomonas aeruginosa. J Immunol 2001; 167: 5880-5886.

9 Fleiszig SM, Zaidi TS, Fletcher EL, Preston MJ, Pier GB. Pseudomonas aeruginosa invades corneal epithelial cells during experimental infection. Infect Immun 1994; 62: 3485-3493.

10 Yanagihara K, Tomono K, Sawai $\mathrm{T}$, et al. Effect of clarithromycin on lymphocytes in chronic respiratory Pseudomonas aeruginosa infection. Am J Respir Crit Care Med 1997; 155: 337-342.

11 Kaneko Y, Yanagihara K, Seki M, et al. Clarithromycin inhibits overproduction of muc5ac core protein in murine model of diffuse panbronchiolitis. Am J Physiol Lung Cell Mol Physiol 2003; 285: L847-L853.

12 Roy-Burman A, Savel RH, Racine S, et al. Type III protein secretion is associated with death in lower respiratory and systemic Pseudomonas aeruginosa infections. J Infect Dis 2001; 183: 1767-1774.

13 Courtney JM, Ennis M, Elborn JS. Cytokines and inflammatory mediators in cystic fibrosis. J Cyst Fibros 2004; 3: 223-231.

14 Bonfield TL, Panuska JR, Konstan MW, et al. Inflammatory cytokines in cystic fibrosis lungs. Am J Respir Crit Care Med 1995; 152: 2111-2118.

15 Mukae H, Kadota J, Ashitani J, et al. Elevated levels of soluble adhesion molecules in serum of patients with diffuse panbronchiolitis. Chest 1997; 112: 1615-1621. 